\title{
Two-sided sampling plan for exponential distribution under type II censored samples
}

\author{
Hacène Belbachir ${ }^{1}$ (D), Mohamed Benahmed ${ }^{* 1,2,3}$ (D) \\ ${ }^{1}$ USTHB, Faculty of Mathematics, RECITS Laboratory, BP 32 El-Alia Bab-Ezzouar 16111, Algiers, \\ Algeria \\ ${ }^{2}$ Higher National School of Statistics and Applied Economics, Koléa 42003, Tipaza, Algeria \\ ${ }^{3}$ CERIST, Research Center on Scientific and Technical Information, Ben Aknoun 16028, Algiers, Algeria
}

\begin{abstract}
Acceptance sampling plan paid the attention of many researchers in the last few years, their works focused basically on Bayesian sampling plans under one-sided decision function and different forms of censoring. In the present paper, a single variable sampling plan for exponential distribution based on type II censored samples under random decision function is developed. For a polynomial loss function, an explicit expression for the Bayes risk is determined. To obtain an approximation for the optimal sampling plan, a simple algorithm based on a discretization method is presented. Finally, an illustrative example and a simulation study followed by extensive tables for the proposed sampling plan are provided.
\end{abstract}

Mathematics Subject Classification (2020). 62D05, 62F15, 62N05

Keywords. Bayesian sampling, type II censored samples, random decision function

\section{Introduction}

Acceptance sampling plan is an important issue for quality control engineers, to determine whether the outcome of products or process are of high quality, the main purpose is to draw the optimal number of items and to determine as much as possible the quality of a batch. Different criteria can be applied for designing sampling plans, such as the decision theory approach and the operating characteristic curve (see e.g. [5, 7]). Decision theory approach is suited to quality control in the sense that the sampling plan is established by making an optimal decision. Extensive researches in different fields have been studied along with this approach, such as $[10,11,18,19]$.

In reliability analysis, the life test experiment is usually censored, which is a random phenomenon. Recently, considerable literature around acceptance sampling plans has been investigated by several authors, including $[9,13,14,16]$ derived the exact distribution of the maximum likelihood estimator (MLE) of the expected lifetime for the case when the lifetime of components follows exponential distribution based on type I and type II

\footnotetext{
*Corresponding Author.

Email addresses: hacenebelbachir@gmail.com (H. Belbachir), benahmed.mohamed@enssea.net (M. Benahmed)

Received: 06.12.2020; Accepted: 17.11.2021
} 
hybrid censored samples. Lin et al. [15] have discussed Bayesian sampling plans under the type I and type II hybrid censoring based on the results of [9]. Chen et al. [8] developed a Bayesian sampling plan for type II censoring based on a curtailed Bayes decision function. Yang et al. [20] proposed a modified type II hybrid censoring such as the life test experiment interrupted at the time $\tau=\min \left\{\max \left(X_{(m)}, t\right), X_{(n)}\right\}$. Prajapati et al. [17] developed a new shrinkage estimator for the expected lifetime of exponential distribution under type I censoring and type I hybrid censoring which always exists even if no failure occurs at the censoring time $\tau=\min \left(X_{(m)}, t\right)$. In addition, Prajapati and his colleagues claimed that the construction of the Bayes decision function (as in [8], which is based on the posterior expectation) is quite difficult, when the loss function is a polynomial with a higher degree or if it is not a polynomial. Balamurali et al. [6] provided a mixed double sampling plan based on process capability index. The case of acceptance sampling plan under nonparametric distribution has been investigate by [3]. Aslam et al. [4] considered the designing of modified multiple dependent state sampling plan under Weibull and Birnbaum-Saunders distributions. Aslam [1,2] have proposed acceptance sampling plan for variable and attribute using the neutrosophic statistics. Işık and Kaya [12] have developed double acceptance sampling plan for Binomial distribution based on neutrosophic statistics. The previous studies have focused on Bayesian sampling plan under one-sided decision function. However, the decision function may be one-sided or two-sided, the first original aspect of the problem is illustrated in Figure 1.

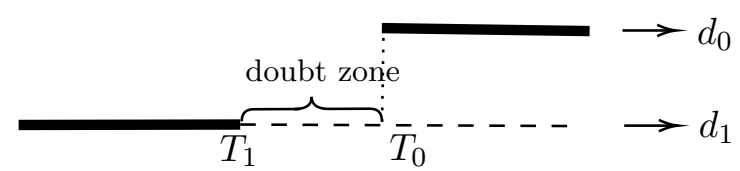

Figure 1. Schematic representation of a decision function with doubt zone.

where $d_{0}$ and $d_{1}$ represent respectively the decisions of accepting and rejecting the batch. $T_{0}$ and $T_{1}$ denote respectively the minimum acceptable and the maximum rejectable surviving time. The doubt zone is a family of regular functions that will be chosen according to the suitability of various models. In the classic case, we pass from $d_{1}$ to $d_{0}$ with a discontinuity i.e. $T_{0}=T_{1}$. Nevertheless, it is crucial to be addressed to the situation when $T_{1}<T_{0}$. With this goal, this paper provides an alternative approach to design a two-sided decision function with a linear doubt zone, such that the transition from $d_{1}$ to $d_{0}$ is done by a linear random function, as shown in Figure 2.

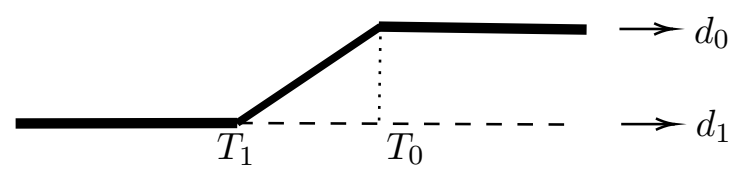

Figure 2. Schematic representation of a decision function with linear doubt zone.

Our aim is to develop a single variable sampling plan when the lifetime of product follows the exponential distribution. The sampling plan is achieved by using a two-sided decision function and under type II censoring. The remainder of this paper is organized as follows. In Section 2, we describe the proposed model and all necessary assumptions. Further, we obtain an explicit expression for the Bayes risk based on a polynomial loss function. In Section 3 we determine an upper bound of the sample size and we suggest an algorithm with an approximation method for finding a local optimal sampling plan. In Section 4, we introduce some numerical results followed by some extensive tables. Finally, Section 5 concludes with a summary. 


\section{Model}

Assume that we have a batch of components presented for life testing. The lifetime of each item is a random variable $X$ which follows a exponential distribution $\operatorname{Exp}(\lambda)$ with expected lifetime $1 / \lambda$ and the probability density function (pdf)

$$
f(x \mid \lambda)= \begin{cases}\lambda \exp (-\lambda x), & \text { for } x \geq 0, \\ 0, & \text { otherwise }\end{cases}
$$

with the scale parameter $\lambda$ is unknown. We suppose also that $\lambda$ has a prior distribution $\Gamma(\alpha, \beta)$, where $\alpha$ and $\beta$ are known, with the pdf

$$
g(\lambda)= \begin{cases}\lambda^{\alpha-1} \exp (-\beta \lambda) \beta^{\alpha} / \Gamma(\alpha), & \text { for } \lambda>0 \\ 0, & \text { otherwise }\end{cases}
$$

A random sample $\left(X_{1}, X_{2}, \ldots, X_{n}\right)$ of size $n$ is taken from a batch for life testing. Let $\left(X_{(1)}, X_{(2)}, \ldots, X_{(n)}\right)$ be the order statistic of $\left(X_{1}, X_{2}, \ldots, X_{n}\right)$. Assume that the type II censoring is adopted, the life test is interrupted after a certain number $m(\leq n)$ of failed items, where we only observe $\underline{X}=\left(X_{(1)}, X_{(2)}, \ldots, X_{(m)}\right)$. Then, the MLE of the expected lifetime $\theta=1 / \lambda$ is given by

$$
\hat{\theta}=\frac{\sum_{i=1}^{m} X_{(i)}+(n-m) X_{(m)}}{m} .
$$

Remark 2.1. $\hat{\theta}$ defined by Equation (2.3) follows a $\Gamma(m, m \lambda)$ distribution.

Note that the proof of Remark 2.1. is existed in the literature. However, to make this paper a self value contain, the following proof is presented:

Let $\left(X_{1}, X_{2}, \ldots, X_{n}\right)$ a sample is selected from $\operatorname{Exp}(\lambda)$, and $\left(X_{(1)}, X_{(2)}, \ldots, X_{(n)}\right)$ is the order statistic, then the joint pdf of $\left(X_{(1)}, X_{(2)}, \ldots, X_{(m)}\right)$ is

$$
g\left(x_{(1)}, x_{(2)}, \ldots, x_{(m)}\right)=\frac{n !}{(n-m) !} \lambda^{m} \exp \left(-\lambda\left[\sum_{i=1}^{m} X_{(i)}+(n-m) X_{(m)}\right]\right) .
$$

According to the transformation $Y_{1}=X_{(1)} Y_{i}=X_{(i)}-X_{(i-1)}$ for $i=2, \ldots, n$ we have

Therefore,

$$
\left\{\begin{array}{l}
X_{(1)}=Y_{1} \\
X_{(2)}=Y_{1}+Y_{2} \\
X_{(3)}=Y_{1}+Y_{2}+Y_{3} \\
\vdots \\
X_{(m)}=Y_{1}+Y_{2}+Y_{3}+\cdots+Y_{m} .
\end{array}\right.
$$

and

$$
\sum_{i=1}^{m} X_{(i)}+(n-m) X_{(m)}=\sum_{i=1}^{m}(n-i+1) Y_{i}
$$

$$
\begin{aligned}
g\left(x_{(1)}, x_{(2)}, \ldots, x_{(m)}\right)=f\left(y_{1}, y_{2}, \ldots, y_{m}\right)=\frac{n !}{(n-m) !} \lambda^{m} \exp \left(-\lambda \sum_{i=1}^{m}(n-i+1) y_{i}\right) \\
=n \lambda e^{-\lambda n y_{1}}(n-1) \lambda e^{-\lambda(n-1) y_{2}} \ldots(n-m+2) \lambda e^{-2 \lambda y_{n-1}}(n-m+1) \lambda e^{-\lambda y_{m}} .
\end{aligned}
$$

Thus, the $Y_{1}, \ldots, Y_{m}$ are mutually independent, such that $Y_{i} \rightsquigarrow \operatorname{Exp}((n-i+1) \lambda)$ for $(i=1, \ldots, m)$, and therefore,

$$
\frac{\sum_{i=1}^{m} X_{(i)}+(n-m) X_{(m)}}{m} \rightsquigarrow \Gamma(m, m \lambda) .
$$

The proof is completed. 


\subsection{Decision role}

Based on the observed data $\underline{x}=\left(x_{(1)}, x_{(2)}, \ldots, x_{(m)}\right)$, a decision function $\delta(\underline{x})$ is made. We consider the following two-sided decision function

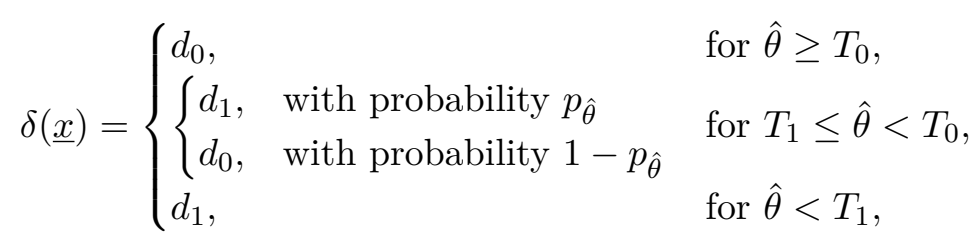

where, $p_{\hat{\theta}}=\frac{T_{0}-\hat{\theta}}{T_{0}-T_{1}}$. The loss to make a decision between $d_{0}$ and $d_{1}$, and select the sampling plan $\left(n, m, T_{0}, T_{1}\right)$ is defined as follows:

$$
L(\lambda, \delta(\underline{x}))= \begin{cases}n C_{s}-(n-m) v_{s}+\tau C_{t}+\sum_{i=0}^{k} a_{i} \lambda^{i}, & \text { if } \delta(\underline{x})=\delta_{0}, \\ n C_{s}-(n-m) v_{s}+\tau C_{t}+C_{r}, & \text { if } \delta(\underline{x})=\delta_{1},\end{cases}
$$

where, the random variable $X_{(m)}$ is the censoring time and $m$ is the number of failure, the parameters $C_{s}, C_{t}$ and $C_{r}$ are positive constants and represent respectively the unit inspection cost, the cost per unit of time used for the test and the loss due to rejection of the batch, the quantity $a_{0}+a_{1} \lambda+\cdots+a_{k} \lambda^{k}$ denotes the loss of accepting the batch and be positive and increasing in $\lambda$. When the life test was finished, the unfailure items can be reused and therefore have the salvage value $v_{s}$, where $0 \leq v_{s}<C_{s}$.

To derive the sampling plan $\left(n, m, T_{0}, T_{1}\right)$ based on the decision function $\delta(\underline{x})$, we carried out the following procedure:

(1) Select a random sample of size $n$ from the batch for life testing based on type II censored samples.

(2) Interrupt the test until the $m$-th failure is observed with $m \leq n$, and record the value of $x_{(1)}, x_{(2)}, \ldots, x_{(m)}$.

(3) Compute the quantity $\hat{\theta}$ as

$$
\hat{\theta}=\frac{\sum_{i=1}^{m} x_{(i)}+(n-m) x_{(m)}}{m} .
$$

(4) Accept the batch if $\hat{\theta} \geq T_{0}$, and reject the batch if $\hat{\theta}<T_{1}$. If $T_{1} \leq \hat{\theta}<T_{0}$ the batch is rejected and accepted with probability $p_{\hat{\theta}}=\left(T_{0}-\hat{\theta}\right) /\left(T_{0}-T_{1}\right)$ and $1-p_{\hat{\theta}}$ respectively.

Theorem 2.2. The Bayes risk can be described by the following equation:

$$
\begin{aligned}
& R\left(n, m, T_{0}, T_{1}\right)=n C_{s}-(n-m) v_{s}+C_{t} \frac{\beta}{\alpha}\left(\begin{array}{c}
n \\
m-1
\end{array}\right) \sum_{j=0}^{m-1}(-1)^{j}\left(\begin{array}{c}
m-1 \\
j
\end{array}\right) \frac{n-m+1}{(n+j-m+1)^{2}}+C_{r} \\
& \quad+\sum_{i=0}^{k} A_{i} \frac{\Gamma(\alpha+i)}{\beta^{i} \Gamma(\alpha)}\left\{1-I_{q_{1}}(m, \alpha+i)-\frac{T_{0}}{T_{0}-T_{1}}\left[I_{q_{0}}(m, \alpha+i)-I_{q_{1}}(m, \alpha+i)\right]\right. \\
& \left.\quad+\frac{\beta}{(\alpha+i-1)\left(T_{0}-T_{1}\right)}\left[I_{q_{0}}(m+1, \alpha+i-1)-I_{q_{1}}(m+1, \alpha+i-1)\right]\right\},
\end{aligned}
$$

where $q_{i}=m T_{i} /\left(m T_{i}+\beta\right)$ for $i=0,1$, and $B_{x}(a, b), I_{x}(a, b)$ denote the incomplete Beta function and the incomplete Beta ratio respectively.

$$
A_{i}= \begin{cases}a_{0}-C_{r}, & \text { for } i=0, \\ a_{i}, & \text { for } i=1, \ldots, k\end{cases}
$$


Proof. Based on the loss function defined in Equation (2.5), the expression of the risk can be computed as follows:

$$
\begin{aligned}
R\left(n, m, T_{0}, T_{1}\right) & =E\{E[L(\lambda, \delta(\underline{x}))]\} \\
& =E\left\{E\left[n C_{s}+C_{t} \tau-(n-m) v_{s}+\left(1-d_{0}\right) C_{r}+d_{0} \sum_{i=0}^{k} a_{i} \lambda^{i} \mid \lambda\right]\right\} \\
& =n C_{s}-(n-m) v_{s}+C_{t} E\left\{E\left[X_{(m)}\right] \mid \lambda\right\}+C_{r}+r\left(n, m \mid d_{0}\right)
\end{aligned}
$$

Such as

$$
\begin{aligned}
& r\left(n, m, \mid d_{0}\right)=E\left\{E\left[d_{0}\left(\sum_{i=0}^{k} a_{i} \lambda^{i}-C_{r}\right) \mid \lambda\right]\right\} \\
& =E\left\{E\left[\sum_{i=0}^{k} A_{i} \lambda^{i}\left(1 \hat{\theta}^{\prime}>T_{1}-p_{\hat{\theta}} 1_{T_{1} \leq \hat{\theta}<T_{0}}\right) \mid \lambda\right]\right\} \\
& =\sum_{i=0}^{k} A_{i} \frac{\beta^{\alpha}}{\Gamma(\alpha)} \int_{0}^{\infty} e^{-\beta \lambda} \lambda^{\alpha+i-1}\left[\int_{0}^{T_{1}} f_{\hat{\theta}}(y) d y+\int_{T_{1}}^{T_{0}} \frac{T_{0}-y}{T_{0}-T_{1}} f_{\hat{\theta}}(y) d y\right] d \lambda \\
& =\sum_{i=0}^{k} A_{i} \int_{0}^{\infty} \frac{m^{m} \beta^{\alpha}}{\Gamma(\alpha) \Gamma(m)} \lambda^{m+\alpha+i-1}\left\{\int_{T_{1}}^{\infty} e^{-(m y+\beta) \lambda} y^{m-1} d y-\int_{T_{1}}^{T_{0}} \frac{T_{0}-y}{T_{0}-T_{1}} e^{-(m y+\beta) \lambda} y^{m-1} d y\right\} d \lambda \\
& =\sum_{i=0}^{k} A_{i} \frac{m^{m} \beta^{\alpha} \Gamma(m+\alpha+i)}{\Gamma(\alpha) \Gamma(m)}\left\{\int_{T_{1}}^{\infty} \frac{y^{m-1}}{(m y+\beta)^{m+\alpha+i}} d y-\int_{T_{1}}^{T_{0}} \frac{T_{0}-y}{T_{0}-T_{1}} \frac{y^{m-1}}{(m y+\beta)^{m+\alpha+i}} d y\right\} .
\end{aligned}
$$

For $z=\frac{m y}{m y+\beta}$, therefore we have

$$
\begin{aligned}
r\left(n, m \mid d_{0}\right)= & \sum_{i=0}^{k} A_{i} \frac{\Gamma(m+\alpha+i)}{\Gamma(\alpha) \Gamma(m) \beta^{i}} \int_{q_{1}}^{1} z^{m-1}(1-z)^{\alpha+i-1} d z-\int_{q_{1}}^{q_{0}} \frac{T_{0}-\frac{\beta z}{m(1-z)}}{T_{0}-T_{1}} z^{m-1}(1-z)^{\alpha+i-1} d z \\
= & \sum_{i=0}^{k} A_{i} \frac{\Gamma(\alpha+i)}{\beta^{i} \Gamma(\alpha)}\left\{1-I_{q_{1}}(m, \alpha+i)-\frac{T_{0}}{T_{0}-T_{1}}\left[I_{q_{0}}(m, \alpha+i)-I_{q_{1}}(m, \alpha+i)\right]\right. \\
& \left.+\frac{\beta}{(\alpha+i-1)\left(T_{0}-T_{1}\right)}\left[I_{q_{0}}(m+1, \alpha+i-1)-I_{q_{1}}(m+1, \alpha+i-1)\right]\right\},
\end{aligned}
$$

and, by a standard computation, the expression of the expected censoring time is given by

$$
E\left\{E\left[X_{(m)}\right] \mid \lambda\right\}=\frac{\beta}{\alpha}\left(\begin{array}{c}
n \\
m-1
\end{array}\right) \sum_{j=0}^{m-1}(-1)^{j}\left(\begin{array}{c}
m-1 \\
j
\end{array}\right) \frac{n-m+1}{(n+j-m+1)^{2}},
$$

therefore

$$
\begin{aligned}
R(n, m, & \left.T_{0}, T_{1}\right)=n C_{s}-(n-m) v_{s}+C_{t} \frac{\beta}{\alpha}\left(\begin{array}{c}
n \\
m-1
\end{array}\right) \sum_{j=0}^{m-1}(-1)^{j}\left(\begin{array}{c}
m-1 \\
j
\end{array}\right) \frac{n-m+1}{(n+j-m+1)^{2}}+C_{r} \\
& +\sum_{i=0}^{k} A_{i} \frac{\Gamma(\alpha+i)}{\beta^{i} \Gamma(\alpha)}\left\{1-I_{q_{1}}(m, \alpha+i)-\frac{T_{0}}{T_{0}-T_{1}}\left[I_{q_{0}}(m, \alpha+i)-I_{q_{1}}(m, \alpha+i)\right]\right. \\
& \left.+\frac{\beta}{(\alpha+i-1)\left(T_{0}-T_{1}\right)}\left[I_{q_{0}}(m+1, \alpha+i-1)-I_{q_{1}}(m+1, \alpha+i-1)\right]\right\} .
\end{aligned}
$$

Thus, the proof is completed.

\section{Algorithm and numerical approximation}

The expression of $R\left(n, m, T_{0}, T_{1}\right)$ is quite complicated, so we cannot evaluate it analytically, based on an approximation method by considering $T_{1}=l T_{0}$, where $0<l<1$, we can obtain local optimal sampling plan numerically. By using the Lagrange polynomials for 
the interpolation points $x_{0}=0.5, x_{1}=1.0$ and $x_{2}=1.5$, the derivative of $R\left(n, m, T_{0}, l T_{0}\right)$ with respect to $T_{0}$ reduces to a quadratic equation which is given by

$$
\sum_{i=0}^{2}\left[h\left(x_{i}\right) \prod_{j=0, j \neq i}^{2} \frac{T_{0}-x_{j}}{x_{i}-x_{j}}\right]=0,
$$

where $h\left(T_{0}\right)=\frac{\partial R\left(n, r, T_{0}, l T_{0}\right)}{\partial T_{0}}$.

\subsection{An upper bound for the optimal sample size}

In order to obtain the optimal sampling plan, we suggest an upper bound for the optimal sample size i.e. we can get a local optimal sampling plan in a finite number of search steps.

Theorem 3.1. The optimal size of the sample is bounded by

$$
N=\min \left\{\left[\frac{C_{r}}{C_{s}-v_{s}}\right],\left[\frac{\sum_{i=0}^{k} a_{i} \gamma_{i}}{C_{s}-v_{s}}\right]\right\},
$$

where $[x]$ is the integer part of $x$, and $\gamma_{i}$ represent the $i$-th moment of $\lambda$.

Proof. Let $(0,0,0,0)$ and $(0,0, \infty, \infty)$ be the sampling plans that accepts and rejects the batch at no sampling case. For $\left(n^{\prime}, m^{\prime}, T_{0}^{\prime}, T_{1}^{\prime}\right)$ an optimal sampling plan, then $R\left(n^{\prime}, m^{\prime}, T_{0}^{\prime}, T_{1}^{\prime}\right) \leq R(0,0,0,0)=\sum_{i=0}^{k} a_{i} \gamma_{i}$. and $R\left(n^{\prime}, m^{\prime}, T_{0}^{\prime}, T_{1}^{\prime}\right) \leq R(0,0, \infty, \infty)=C_{r}$. As $n\left(C_{s}-v_{s}\right) \leq R\left(n^{\prime}, m^{\prime}, T_{0}^{\prime}, T_{1}^{\prime}\right)$, therefore we have

$$
\begin{aligned}
n\left(C_{s}-v_{s}\right) & \leq \min \left\{C_{r}, \sum_{i=0}^{k} a_{i} \gamma_{i}\right\} \\
n & \leq \min \left\{\left[\frac{C_{r}}{C_{s}-v_{s}}\right],\left[\frac{\sum_{i=0}^{k} a_{i} \gamma_{i}}{C_{s}-v_{s}}\right]\right\} .
\end{aligned}
$$

Thus, the proof is completed.

\subsection{Derivation of the optimal sampling plan}

In this subsection we determine local optimal sampling plans for two situations, when $T_{1}$ is fixed in the interval $\left(0, T_{0}\right)$ for fixed $l$, we denote its related local optimal sampling plan by $\left(n_{0}, m_{0}, T_{0}, T_{1}\right)$, and when $T_{1}$ is flexible in the interval $\left(0, T_{0}\right)$, the correspondent local optimal sampling plan is $\left(n_{B}, m_{B}, T_{0}, T_{1}\right)$. To derive the local optimal sampling plan under the two situations, the following two schemes are proposed

Scheme 1:

(1) Start with $(n, m)=(0,0)$, compute $N$ from Equation (3.2), and compute $R\left(0,0, T_{0}(n, m), T_{1}(n, m)\right)=\min \left\{R(0,0, \infty, \infty)=C_{r}, R(0,0,0,0)=\sum_{i=0}^{k} a_{i} \gamma_{i}\right\}$.

(2) For each $n=1, \ldots, N$ and $m=1, \ldots, n$, compute the optimal $T_{0}(n, m)$ and $T_{1}(n, m)=l T_{0}(n, m)$ such that $T_{0}(n, m)=\min _{1 \leq i \leq 2}\left\{R\left(n, m, T_{0}^{(i)}, l T_{0}^{(i)}\right) \mid T_{0}^{(i)}>0\right\}$, $T_{0}^{(i)}$ is the i-th solution of Equation (3.1).

(3) By comparison, choose $\left(n_{0}, m_{0}, T_{0}, T_{1}\right)$ which corresponds to the smallest value of the Bayes risks $R\left(n, m, T_{0}(n, m), T_{1}(n, m)\right)$.

Scheme 2:

(1) Start with $(n, m)=(0,0)$, compute $N$ from Equation (3.2), and compute $R\left(0,0, T_{0}(n, m), T_{1}(n, m)\right)=\min \left\{R(0,0, \infty, \infty)=C_{r}, R(0,0,0,0)=\sum_{i=0}^{k} a_{i} \gamma_{i}\right\}$. 
(2) For each $n=1, \ldots, N$ and $m=1, \ldots, n$, compute the optimal $T_{0}(n, m)$ such that $T_{0}(n, m)=\min _{1 \leq i \leq 2}\left\{R\left(n, m, T_{0}^{(i)}, l T_{0}^{(i)}\right) \mid T_{0}^{(i)}>0\right\}, T_{0}^{(i)}$ is the $\mathrm{i}$-th solution Equation (3.1). Compute $T_{1}(n, m)$ using grid search method with grid size $T_{0}(n, m) / 1000$.

(3) By comparison, choose $\left(n_{B}, m_{B}, T_{0}, T_{1}\right)$ which corresponds to the smallest value of the Bayes risks $R\left(n, m, T_{0}(n, m), T_{1}(n, m)\right)$.

\section{Numerical results}

To illustrate the proposed model, we assume that the loss is a quadratic function with $(k=2)$. Various numerical examples are depicted in Tables 1-6. In each table we denote $\left(n_{B}, m_{B}, T_{0}, T_{1}\right) \equiv S_{B}$ and $\left(n_{0}, m_{0}, T_{0}, T_{1}\right) \equiv S_{0}$, and their minimum Bayes risk respectively $R\left(n_{B}, m_{B}, T_{0}, T_{1}\right) \equiv R_{B}$ and $R\left(n_{0}, m_{0}, T_{0}, T_{1}\right) \equiv R_{0}$. To examine the behavior of the Bayes risk function, we vary one(two) parameter(s) or coefficient(s) while the others keep fixed. So, as the true values of parameters and coefficients of the model for which we made the calculations, we take: $\alpha=2.5, \beta=1, a_{0}=a_{1}=a_{2}=5, C_{s}=0.5, v_{s}=0.2$, $C_{t}=2$ and $C_{r}=50$. For the sampling plan $\left(n_{0}, m_{0}, T_{0}, T_{1}\right)$ we take $l=2 / 3$. Furher, the standard values are indicated by '*', and the corresponding local optimal sampling plan and the minimum Bayes risk are in bold. The efficiency values in Tables 1-6 defined by eff $=100 R_{0} / R_{B}$ is the ratio of the minimum Bayes risks $R_{0}$ and $R_{B}$.

The local optimal sampling plan for the standard values of parameters and coefficients mentioned above for $\left(n_{B}, m_{B}, T_{0}, T_{1}\right)=(6,4,0.4860,0.3611)$, which means: we put 6 items for life testing and the life test terminates after the 4 -th failure. We may accept the batch if the estimator of the average lifetime $\hat{\theta}$ is greater than 0.4860 . For $\hat{\theta}$ is between 0.4860 and 0.3611 , the batch is rejected and accepted with probability $p_{\hat{\theta}}=\frac{0.4860-\hat{\theta}}{0.4860-0.3611}$ and $1-p_{\hat{\theta}}$, with the Byes risk $R_{B}=43.1853$. Further, corresponding to $\left(\alpha, \beta, a_{0}, a_{1}, a_{2}, C_{s}, v_{s}, C_{t}, C_{r}\right)=(2.5,1,5,5,5,0.5,0.2,2,50)$, the local optimal sampling plan $\left(n_{0}, m_{0}, T_{0}, T_{1}\right)$ is given by $(6,4,0.5638,0.3759)$, the correspondent Bayes risk is $R_{0}=43.3182$.

Table 1. Local optimal sampling plans and their minimum Bayes risks for $\alpha$ and $\beta$ vary.

\begin{tabular}{ccccccccccccc}
\hline$\alpha$ & $\beta$ & $n_{B}$ & $m_{B}$ & $T_{0}$ & $T_{1}$ & $R_{B}$ & $n_{0}$ & $m_{0}$ & $T_{0}$ & $T_{1}$ & $R_{0}$ & eff(\%) \\
\hline 1.5 & 0.2 & 2 & 1 & 0.9941 & 0.9135 & 49.4396 & 3 & 2 & 0.9495 & 0.6330 & 49.4692 & 100.06 \\
1.5 & 0.4 & 5 & 3 & 0.5381 & 0.5375 & 45.3279 & 5 & 3 & 0.7794 & 0.5196 & 45.8101 & 101.06 \\
1.5 & 0.6 & 6 & 3 & 0.4883 & 0.3862 & 40.5936 & 7 & 4 & 0.5702 & 0.3801 & 40.7736 & 100.44 \\
1.5 & 0.8 & 7 & 3 & 0.4532 & 0.2624 & 36.1097 & 7 & 3 & 0.3671 & 0.2448 & 36.3404 & 100.64 \\
2.0 & 0.2 & 0 & 0 & $\infty$ & $\infty$ & 50.0000 & 0 & 0 & $\infty$ & $\infty$ & 50.0000 & 100.00 \\
2.0 & 0.4 & 3 & 2 & 0.9503 & 0.6063 & 49.2325 & 3 & 2 & 0.9425 & 0.6284 & 49.2339 & 100.00 \\
2.0 & 0.6 & 6 & 4 & 0.4974 & 0.4591 & 45.8774 & 5 & 3 & 0.7731 & 0.5154 & 46.3920 & 101.12 \\
2.0 & 0.8 & 7 & 4 & 0.4864 & 0.3629 & 42.0000 & 7 & 4 & 0.5670 & 0.3780 & 42.1410 & 100.34 \\
0.0 & 0.0 & 0 & 0 & $\infty$ & $\infty$ & 50.0000 & 0 & 0 & $\infty$ & $\infty$ & 50.0000 & 100.00 \\
2.5 & 0.6 & 3 & 2 & 0.9425 & 0.6088 & 49.3219 & 3 & 2 & 0.9367 & 0.6245 & 49.3227 & 100.00 \\
2.5 & 0.8 & 6 & 4 & 0.4977 & 0.4579 & 46.4831 & 5 & 3 & 0.7676 & 0.5118 & 47.0093 & 101.13 \\
$\mathbf{2 . 5}$ & $\mathbf{1 . 0}$ & $\mathbf{6}$ & $\mathbf{4}$ & $\mathbf{0 . 4 8 6 0}$ & $\mathbf{0 . 3 6 1 1}$ & $\mathbf{4 3 . 1 8 5 3}$ & $\mathbf{6}$ & $\mathbf{4}$ & $\mathbf{0 . 5 6 3 8}$ & $\mathbf{0 . 3 7 5 9}$ & $\mathbf{4 3 . 3 1 8 2}$ & $\mathbf{1 0 0 . 3}$ \\
3.0 & 0.8 & 3 & 2 & 0.9355 & 0.6109 & 49.4788 & 3 & 2 & 0.9316 & 0.6211 & 49.4792 & 100.00 \\
3.0 & 1.0 & 6 & 4 & 0.4979 & 0.4565 & 47.0288 & 4 & 3 & 0.7628 & 0.5085 & 47.5362 & 101.08 \\
3.0 & 1.2 & 6 & 4 & 0.4867 & 0.3597 & 44.1567 & 6 & 4 & 0.5608 & 0.3738 & 44.2800 & 100.28 \\
0.0 & 0.0 & 0 & 0 & $\infty$ & $\infty$ & 50.0000 & 0 & 0 & $\infty$ & $\infty$ & 50.0000 & 100.00 \\
3.5 & 1.0 & 3 & 2 & 0.9294 & 0.6125 & 49.6417 & 3 & 2 & 0.9271 & 0.6181 & 49.6418 & 100.00 \\
3.5 & 1.2 & 6 & 4 & 0.4980 & 0.4552 & 47.5008 & 4 & 3 & 0.7585 & 0.5057 & 47.9645 & 100.98 \\
\hline
\end{tabular}


In Table 1 , it is easy to see that $R_{B}$ is less than $R_{0}$ for each variation of $(\alpha, \beta)$ and we observe that the difference between $R_{B}$ and $R_{0}$ becomes small for $\alpha$ is fixed and $\beta$ decreases. Further, for $(\alpha, \beta)=(2.0,0.2),(2.5,0.4),(3.5,0.8)$, the batch is rejected without take any sample cost, with $S_{B}=S_{0}=(0,0, \infty, \infty)$ and $R_{B}=R_{0}=50$. On the other hand, $n_{B}$ and $m_{B}$ in most cases are greater than or equal to $n_{0}$ and $m_{0}$ respectively, so by the sampling plan $\left(n_{B}, m_{B}, T_{0}, T_{1}\right)$ we can observe more information about the expected lifetime $1 / \lambda$ of the items set in life testing. So, based on these data, the decision function $\delta(\underline{x})$ can be an appropriate decision with a smaller risk. In Tables 2-4, we observe that, the Bayes risk for both cases is nondecreasing in $a_{0}, a_{1}$ and $a_{2}$. As expected, in each table, we can see that $R_{B}$ is always less than $R_{0}$, while in most cases the efficiency values are close to 1 and in general less than $101 \%$. Furthermore, there are some cases where the sampling plans $S_{B}$ and $S_{0}$ occur at no sampling case, as can be seen from Table 4 , when $a_{2}=2$, the optimal sampling plans take the form $(0,0,0,0)$, which means the batch is accepted without take any sample cost with the Bayes risk $R_{B}=R_{0}=35$. In Table 5 , it can be seen that, the number of observed failures is equal to the optimal sample size when $C_{t}$ closes to 0 . Otherwise, when $C_{t}$ increases, the number of observed failures decreases and the optimal sample size increases, and this is due to the effect of $C_{t}$ value on behavior of the Bayes risk. In Table 6, as expected the Bayes risk of $S_{B}$ and $S_{0}$ is increasing when $C_{r}$ increase with $R_{B}<R_{0}$.

Table 2. Local optimal sampling plans and their minimum Bayes risks for $a_{0}$ varies.

\begin{tabular}{lccccccccccc}
\hline$a_{0}$ & $n_{B}$ & $m_{B}$ & $T_{0}$ & $T_{1}$ & $R_{B}$ & $n_{0}$ & $m_{0}$ & $T_{0}$ & $T_{1}$ & $R_{0}$ & eff(\%) \\
\hline 0 & 6 & 4 & 0.4736 & 0.2884 & 40.7881 & 5 & 3 & 0.4317 & 0.2878 & 41.0116 & 100.55 \\
1 & 6 & 4 & 0.4770 & 0.3020 & 41.2778 & 6 & 4 & 0.4348 & 0.2899 & 41.3476 & 100.17 \\
2 & 6 & 4 & 0.4792 & 0.3158 & 41.7626 & 6 & 4 & 0.4700 & 0.3133 & 41.7655 & 100.01 \\
3 & 6 & 4 & 0.4813 & 0.3302 & 42.2422 & 6 & 4 & 0.5031 & 0.3354 & 42.2557 & 100.03 \\
4 & 6 & 4 & 0.4847 & 0.3456 & 42.7165 & 6 & 4 & 0.5343 & 0.3562 & 42.7810 & 100.15 \\
$\mathbf{5}^{*}$ & $\mathbf{6}$ & $\mathbf{4}$ & $\mathbf{0 . 4 8 6 0}$ & $\mathbf{0 . 3 6 1 1}$ & $\mathbf{4 3 . 1 8 5 3}$ & $\mathbf{6}$ & $\mathbf{4}$ & $\mathbf{0 . 5 6 3 8}$ & $\mathbf{0 . 3 7 5 9}$ & $\mathbf{4 3 . 3 1 8 2}$ & $\mathbf{1 0 0 . 3 1}$ \\
6 & 6 & 4 & 0.4889 & 0.3779 & 43.6483 & 6 & 4 & 0.5918 & 0.3945 & 43.8531 & 100.47 \\
8 & 6 & 4 & 0.4931 & 0.4137 & 44.5566 & 6 & 4 & 0.6435 & 0.4290 & 44.8853 & 100.74 \\
10 & 5 & 3 & 0.4988 & 0.4898 & 45.4289 & 5 & 3 & 0.7155 & 0.4770 & 45.7791 & 100.77 \\
15 & 5 & 3 & 0.6685 & 0.6678 & 47.5853 & 4 & 2 & 0.8579 & 0.5720 & 47.6792 & 100.20 \\
20 & 4 & 2 & 0.9183 & 0.6897 & 49.0483 & 4 & 2 & 0.9443 & 0.6295 & 49.0575 & 100.02 \\
25 & 0 & 0 & $\infty$ & $\infty$ & $\infty$ & 0 & 0 & $\infty$ & $\infty$ & 50.0000 & 100.00 \\
\hline
\end{tabular}

Table 3. Local optimal sampling plans and their minimum Bayes risks for $a_{1}$ varies.

\begin{tabular}{lccccccccccc}
\hline$a_{1}$ & $n_{B}$ & $m_{B}$ & $T_{0}$ & $T_{1}$ & $R_{B}$ & $n_{0}$ & $m_{0}$ & $T_{0}$ & $T_{1}$ & $R_{0}$ & eff $(\%)$ \\
\hline 0 & 6 & 4 & 0.4429 & 0.1873 & 39.2967 & 2 & 1 & 0.1493 & 0.0995 & 43.7406 & 111.31 \\
1 & 6 & 4 & 0.4559 & 0.2197 & 40.1363 & 2 & 1 & 0.3334 & 0.2222 & 42.7147 & 106.42 \\
2 & 6 & 4 & 0.4660 & 0.2530 & 40.9466 & 4 & 2 & 0.3758 & 0.2505 & 41.8472 & 102.20 \\
3 & 6 & 4 & 0.4735 & 0.2874 & 41.7254 & 5 & 3 & 0.4300 & 0.2867 & 41.8943 & 100.40 \\
4 & 6 & 4 & 0.4806 & 0.3235 & 42.4716 & 6 & 4 & 0.4880 & 0.3253 & 42.4733 & 100.00 \\
$\mathbf{5}^{*}$ & $\mathbf{6}$ & $\mathbf{4}$ & $\mathbf{0 . 4 8 6 0}$ & $\mathbf{0 . 3 6 1 1}$ & $\mathbf{4 3 . 1 8 5 3}$ & $\mathbf{6}$ & $\mathbf{4}$ & $\mathbf{0 . 5 6 3 8}$ & $\mathbf{0 . 3 7 5 9}$ & $\mathbf{4 3 . 3 1 8 2}$ & $\mathbf{1 0 0 . 3 1}$ \\
6 & 6 & 4 & 0.4915 & 0.4011 & 43.8664 & 6 & 4 & 0.6259 & 0.4173 & 44.1644 & 100.68 \\
8 & 6 & 4 & 0.4989 & 0.4875 & 45.1330 & 5 & 3 & 0.7454 & 0.4970 & 45.5426 & 100.91 \\
10 & 6 & 4 & 0.6242 & 0.6236 & 46.4597 & 5 & 3 & 0.8163 & 0.5442 & 46.5692 & 100.24 \\
15 & 4 & 2 & 0.9444 & 0.7546 & 48.3618 & 4 & 2 & 0.9831 & 0.6554 & 48.3910 & 100.06 \\
20 & 4 & 2 & 0.9944 & 0.9606 & 49.4869 & 4 & 2 & 1.0698 & 0.7132 & 49.6149 & 100.26 \\
25 & 0 & 0 & $\infty$ & $\infty$ & 50.0000 & 0 & 0 & $\infty$ & $\infty$ & 50.0000 & 100.00 \\
\hline
\end{tabular}


Table 4. Local optimal sampling plans and their minimum Bayes risks for $a_{2}$ varies.

\begin{tabular}{lccccccccccc}
\hline$a_{2}$ & $n_{B}$ & $m_{B}$ & $T_{0}$ & $T_{1}$ & $R_{B}$ & $n_{0}$ & $m_{0}$ & $T_{0}$ & $T_{1}$ & $R_{0}$ & eff $(\%)$ \\
\hline 2 & 0 & 0 & 0.0000 & 0.0000 & 35.0000 & 0 & 0 & 0.0000 & 0.0000 & 35.0000 & 100.00 \\
3 & 5 & 3 & 0.3906 & 0.1160 & 39.8685 & 0 & 0 & 0.0000 & 0.0000 & 43.7500 & 109.74 \\
4 & 6 & 4 & 0.4637 & 0.2513 & 41.6877 & 4 & 2 & 0.3617 & 0.2411 & 42.4664 & 101.87 \\
$\mathbf{5}^{*}$ & $\mathbf{6}$ & $\mathbf{4}$ & $\mathbf{0 . 4 8 6 0}$ & $\mathbf{0 . 3 6 1 1}$ & $\mathbf{4 3 . 1 8 5 3}$ & $\mathbf{6}$ & $\mathbf{4}$ & $\mathbf{0 . 5 6 3 8}$ & $\mathbf{0 . 3 7 5 9}$ & $\mathbf{4 3 . 3 1 8 2}$ & $\mathbf{1 0 0 . 3 1}$ \\
6 & 6 & 4 & 0.4979 & 0.4675 & 44.4318 & 6 & 4 & 0.7057 & 0.4705 & 44.9243 & 101.11 \\
7 & 6 & 4 & 0.6141 & 0.6135 & 45.6829 & 5 & 3 & 0.8154 & 0.5436 & 45.9398 & 100.56 \\
8 & 5 & 3 & 0.8648 & 0.5976 & 46.6689 & 5 & 3 & 0.8732 & 0.5822 & 46.6700 & 100.00 \\
9 & 5 & 3 & 0.9065 & 0.6309 & 47.2430 & 5 & 3 & 0.9157 & 0.6105 & 47.2452 & 100.00 \\
10 & 5 & 3 & 0.9327 & 0.6725 & 47.7162 & 5 & 3 & 0.9484 & 0.6323 & 47.7250 & 100.02 \\
12 & 5 & 3 & 0.9646 & 0.7630 & 48.4671 & 5 & 3 & 0.9960 & 0.6640 & 48.5151 & 100.10 \\
15 & 5 & 3 & 0.9899 & 0.9028 & 49.2940 & 5 & 3 & 1.0425 & 0.6950 & 49.4732 & 100.36 \\
20 & 0 & 0 & $\infty$ & $\infty$ & 50.0000 & 0 & 0 & $\infty$ & $\infty$ & 50.0000 & 100.00 \\
\hline
\end{tabular}

Table 5. Local optimal sampling plans and their minimum Bayes risks for $C_{t}$ varies.

\begin{tabular}{lccccccccccc}
\hline$C_{t}$ & $n_{B}$ & $m_{B}$ & $T_{0}$ & $T_{1}$ & $R_{B}$ & $n_{0}$ & $m_{0}$ & $T_{0}$ & $T_{1}$ & $R_{0}$ & eff(\%) \\
\hline 0.1 & 5 & 5 & 0.4859 & 0.3489 & 41.4131 & 5 & 5 & 0.5436 & 0.3624 & 41.5049 & 100.22 \\
0.5 & 4 & 4 & 0.4860 & 0.3611 & 42.0130 & 5 & 5 & 0.5436 & 0.3624 & 42.1138 & 100.24 \\
1.0 & 5 & 4 & 0.4860 & 0.3611 & 42.4742 & 5 & 4 & 0.5638 & 0.3759 & 42.6071 & 100.31 \\
1.5 & 6 & 4 & 0.4860 & 0.3611 & 42.8686 & 6 & 4 & 0.5638 & 0.3759 & 43.0015 & 100.31 \\
$\mathbf{2 . 0}$ & $\mathbf{6}$ & $\mathbf{4}$ & $\mathbf{0 . 4 8 6 0}$ & $\mathbf{0 . 3 6 1 1}$ & $\mathbf{4 3 . 1 8 5 3}$ & $\mathbf{6}$ & $\mathbf{4}$ & $\mathbf{0 . 5 6 3 8}$ & $\mathbf{0 . 3 7 5 9}$ & $\mathbf{4 3 . 3 1 8 2}$ & $\mathbf{1 0 0 . 3 1}$ \\
2.5 & 7 & 4 & 0.4860 & 0.3611 & 43.4845 & 7 & 4 & 0.5638 & 0.3759 & 43.6174 & 100.31 \\
3.0 & 7 & 4 & 0.4860 & 0.3611 & 43.7377 & 7 & 4 & 0.5638 & 0.3759 & 43.8706 & 100.30 \\
4.0 & 6 & 3 & 0.4878 & 0.3824 & 44.1684 & 6 & 3 & 0.5919 & 0.3946 & 44.3425 & 100.39 \\
5.0 & 7 & 3 & 0.4878 & 0.3824 & 44.5223 & 7 & 3 & 0.5919 & 0.3946 & 44.6965 & 100.39 \\
10.0 & 9 & 3 & 0.4878 & 0.3824 & 45.9504 & 9 & 3 & 0.5919 & 0.3946 & 46.1245 & 100.38 \\
15.0 & 9 & 2 & 0.4910 & 0.4261 & 46.8565 & 9 & 2 & 0.6359 & 0.4239 & 47.0473 & 100.41 \\
20.0 & 10 & 2 & 0.4910 & 0.4261 & 47.6102 & 10 & 2 & 0.6359 & 0.4239 & 47.8010 & 100.40 \\
\hline
\end{tabular}

Table 6. Local optimal sampling plans and their minimum Bayes risks for $C_{r}$ varies.

\begin{tabular}{cccccccccccc}
\hline$C_{r}$ & $n_{B}$ & $m_{B}$ & $T_{0}$ & $T_{1}$ & $R_{B}$ & $n_{0}$ & $m_{0}$ & $T_{0}$ & $T_{1}$ & $R_{0}$ & $\mathrm{eff}(\%)$ \\
\hline 30 & 0 & 0 & $\infty$ & $\infty$ & 30.0000 & 0 & 0 & $\infty$ & $\infty$ & 30.0000 & 100.00 \\
35 & 4 & 2 & 0.9183 & 0.6897 & 34.0483 & 4 & 2 & 0.9443 & 0.6295 & 34.0575 & 100.03 \\
40 & 5 & 3 & 0.6685 & 0.6678 & 37.5853 & 4 & 2 & 0.8579 & 0.5720 & 37.6792 & 100.25 \\
45 & 5 & 3 & 0.4988 & 0.4898 & 40.4289 & 5 & 3 & 0.7155 & 0.4770 & 40.7791 & 100.87 \\
$\mathbf{5 0}$ & $\mathbf{6}$ & $\mathbf{4}$ & $\mathbf{0 . 4 8 6 0}$ & $\mathbf{0 . 3 6 1 1}$ & $\mathbf{4 3 . 1 8 5 3}$ & $\mathbf{6}$ & $\mathbf{4}$ & $\mathbf{0 . 5 6 3 8}$ & $\mathbf{0 . 3 7 5 9}$ & $\mathbf{4 3 . 3 1 8 2}$ & $\mathbf{1 0 0 . 3 1}$ \\
55 & 6 & 4 & 0.4736 & 0.2884 & 45.7881 & 5 & 3 & 0.4317 & 0.2878 & 46.0116 & 100.49 \\
60 & 6 & 4 & 0.4574 & 0.2282 & 48.2709 & 4 & 2 & 0.2697 & 0.1798 & 50.7146 & 105.06 \\
65 & 7 & 5 & 0.4404 & 0.1797 & 50.6478 & 2 & 1 & 0.0229 & 0.0153 & 61.1168 & 120.67 \\
70 & 7 & 5 & 0.4129 & 0.1362 & 52.9360 & 0 & 0 & 0.0000 & 0.0000 & 61.2500 & 115.71 \\
75 & 7 & 5 & 0.3739 & 0.0946 & 55.2441 & 0 & 0 & 0.0000 & 0.0000 & 61.2500 & 110.87 \\
80 & 8 & 6 & 0.3169 & 0.0621 & 57.9032 & 0 & 0 & 0.0000 & 0.0000 & 61.2500 & 105.78 \\
85 & 0 & 0 & 0.0000 & 0.0000 & 61.2500 & 0 & 0 & 0.0000 & 0.0000 & 61.2500 & 100.00 \\
\hline
\end{tabular}

\section{Conclusion}

With the Bayesian approach, we have determined single sampling plans, for exponential distribution with type II censoring, we proposed a two-sided decision function with a random doubt zone for which we suggest an approach to decide the quality of a batch with 
a suitable probability. Explicit expression for the Bayes risk is obtained for the polynomial loss function which includes the unit inspection cost, the time-cost, the rejection cost, the salvage value and the after-sales cost. To evaluate the Bayes risk, we assume that the loss function is a quadratic function, such as the computations can be done in a similar way for higher degree. Furthermore, after determining an upper bound for the optimal size of sample, we developed a finite algorithm which allowed to simulate the risk function numerically based on the grid search method. Also we introduce a comparison performance and some optimal sampling plans followed by related Bayes risks for different values of parameters. From the numerical presented above, we can see that the Bayes risk based on the proposed random decision function have robust behavior with considering to changes in the parameters and coefficients in the proposed sampling plan.

In some real cases, the quality characteristics data is derived from a complex process or from an uncertain environment, so the use of the neutrosophic statistics can model the uncertainty and handle the human's assessments. To sum up, the current study can be extended using the neutrosophic statistics based on appropriate approach for the doubt zone in the decision function.

\section{Acknowledgment}

We are grateful to the Editor and the two referees for their careful reading of the manuscript, their insightful suggestions and for their comments as well that greatly improved the presentation of this work. The paper is partially supported by DGRSDT Grant CD656701 in Algeria.

\section{References}

[1] M. Aslam, A variable acceptance sampling plan under neutrosophic statistical interval method, Symmetry 11 (1), 114, 2019.

[2] M. Aslam, A new attribute sampling plan using neutrosophic statistical interval method, Complex Intell. Systems 5 (4), 365370, 2019.

[3] M. Aslam, Product acceptance determinations using new nonparametric sign sampling plan, Int. J. Stat. Manag. Syst. 23 (8), 1561-1570, 2020.

[4] M. Aslam, P. Jeyadurga, S. Balamurali, M. Azam and A. AL-Marshadi, Economic determination of modified multiple dependent state sampling plan under some lifetime distributions, J. Math., Doi:10.1155/2021/7470196, 2021.

[5] M. Aslam, M.A. Raza and L. Ahmad, Acceptance sampling plans for two-stage process for multiple manufacturing lines under neutrosophic statistics, J. Intell. Fuzzy Syst. 37 (6), 7839-7850, 2019.

[6] S. Balamurali, M. Aslam, L. Ahmad and C.H. Jun, A mixed double sampling plan based on $C_{p k}$, Comm. Statist. Theory Methods Communications 49 (8), 1840-1857, 2019.

[7] R. Bhattacharya and M. Aslam, Generalized multiple dependent state sampling plans in presence of measurement data, IEEE Access 8, 162775-162784, 2020.

[8] L.S. Chen, M.C. Yang and T. Liang, Curtailed Bayesian sampling plans for exponential distributions based on Type-II censored samples, J. Stat. Comput. Simul. 87 (6), 1160-1178, 2017.

[9] A. Childs, B. Chandrasekar, N. Balakrishnan and D. Kundu, Exact likelihood inference based on type-I and type-II hybrid censored samples from the exponential distribution, Ann. Inst. Statist. Math. 55 (2), 319-330, 2003.

[10] W.C. Guenther, On the determination of single sampling attribute plans based upon a linear cost model and a prior distribution, Technometrics 13 (3), 483-498, 1971.

[11] A. Hald, Asymptotic properties of Bayesian single sampling plans, J. R. Stat. Soc. Ser. B. Stat. Methodol. 29 (1), 162-173, 1968. 
[12] G. Işı and İ. Kaya, Effects of neutrosophic binomial distribution on double acceptance sampling plan, Conference Proceeding Science and Technology 3 (1), 68-76, 2020.

[13] Y. Lam, An optimal single variable sampling plan with censoring, The Statistician 39 (1), 53-67, 1990.

[14] Y. Lam, Bayesian variable sampling plans for the exponential distribution with type I censoring, Ann. Statist. 22 (2), 696-711, 1994.

[15] C.T. Lin, Y.L. Huang and N. Balakrishnan, Exact Bayesian variable sampling plans for the exponential distribution based on type-I and type-II hybrid censored samples, Comm. Statist. Simulation Comput. 37 (6), 1101-1116, 2008.

[16] Y.P Lin, L. Tachen and W.T. Huang, Bayesian sampling plans for exponential distribution based on the type I censoring data, Ann. Inst. Statist. Math. 54 (1), 100-113, 2002.

[17] D. Prajapati, S. Mitra and D. Kundu, A new decision theoretic sampling plan for exponential distribution under type-I censoring, Comm. Statist. Simulation Comput. 49 (2), 453-471, 2020.

[18] G.B. Wetherill, Sampling Inspection and Quality Control, 2nd ed., Chapman and Hall, London, 1977.

[19] G.B. Wetherill and G.E.G. Campling, The decision theory approach to sampling inspection, J. R. Stat. Soc. Ser. B. Stat. Methodol. 28 (3), 381-416, 1966.

[20] M.C. Yang, L.S. Chen and T. Liang, Optimal Bayesian variable sampling plans for exponential distributions based on modified type-II hybrid censored samples, Comm. Statist. Simulation Comput. 46 (6), 4722-4744, 2017. 